\title{
EFECTO DE LA TEMPERATURA DE EXTRUSIÓN EN LA MEZCLA DE HARINAS DE TARWI (Lupinus mutabilis) Y ARROZ (Orysa sativa) PARA LA PRODUCCIÓN DE UN SNACK
}

\section{Effect of extrusion temperature on the mixture of tarwi (Lupinus mutabilis) and rice (Orysa sativa) flours for the production of a snack}

$\begin{array}{lll}\text { (iD) Jair Jol Aranda Tarazona }{ }^{1} & \text { (iD Glimer Idan Bocanegra Reyes }{ }^{1} & \text { iD Lucia Pantoja-Tirado }{ }^{2} \\ \begin{array}{lll}\text { iD Gino Paul Prieto Rosales }{ }^{2} & \text { (iD) Gilbert Rodriguez-Paucar }{ }^{1} & \text { (iD Elza Berta Aguirre Vargas }{ }^{1}\end{array}\end{array}$

'Universidad Nacional del Santa, Perú.

2Universidad Nacional Autónoma de Tayacaja Daniel Hernández Morillo, Perú.

Correspondencia:

Dra. Elza Aguirre Vargas

eaguirrev@gmail.com

\section{RESUMEN}

Los granos de tarwi (Lupinus mutabilis S.) poseen un elevado contenido proteico, siendo además muy ricos en algunos aminoácidos esenciales, tales como la lisina. Sin embargo, los parámetros de extrusión afectan de manera significativa los contenidos proteicos de los productos generados. El objetivo principal del estudio fue determinar la mejor temperatura de extrusión en un snack elaborado a partir de harinas de tarwi y arroz. Para la producción del snack se utilizó la harina de tarwi (Lupinus mutabilis Sweet), en tres concentraciones $(8,10$ y $12 \%)$ y tres proporciones de la harina de arroz (Orysa sativa) ( 88,90 y $92 \%)$. Los parámetros de extrusión fueron: temperatura de extrusión $(100,110,115)^{\circ} \mathrm{C}$, velocidad de rotación del tornillo $1560 \mathrm{rpm}$, velocidad de alimentación 1 kg/min, velocidad de cuchilla $630 \mathrm{rpm}$, caudal de agua $19.5 \mathrm{ml} / \mathrm{min}$ y diámetro de la boquilla de salida 0,6 mm. Se utilizó un diseño factorial multinivel, consiguiendo 9 corridas experimentales. Los resultados de los análisis sensorial y químico proximal determinaron que la mejor formulación del snack se obtuvo con el $90 \%$ del contenido de arroz y $10 \%$ del contenido de tarwi; además se determinó que la temperatura de extrusión fue de $110^{\circ} \mathrm{C}$. El snack elaborado resultó con un contenido proteico de $13.11 \%, 4.03 \%$ de fibra y su tiempo de vida útil fue de 25 días; resultando un producto de alto contenido proteico, de alta aceptación organoléptica y apto para el consumo.

Palabras clave: Harina de tarwi, harina de arroz, extrusión, proteínas, vida útil.

\section{ABSTRACT}

Tarwi beans (Lupinus mutabilis S.) have a high protein content and are also very rich in some essential amino acids, such as lysine. However, extrusion parameters significantly affect the protein content of the products generated. The main objective of the study 
was to determine the best extrusion temperature for a snack made from tarwi and rice flours. For the production of the snack, tarwi flour (Lupinus mutabilis S.) was used in three concentrations ( 8,10 and $12 \%$ ) and three proportions of rice flour (Orysa sativa) (88, 90 and 92\%). The extrusion parameters were: extrusion temperature $(100,110,115)$ ${ }^{\circ} \mathrm{C}$, screw rotation speed $1560 \mathrm{rpm}$, feed speed $1 \mathrm{~kg} / \mathrm{min}$, knife speed $630 \mathrm{rpm}$, water flow rate $19.5 \mathrm{ml} / \mathrm{min}$ and outlet nozzle diameter $0.6 \mathrm{~mm}$. A multilevel factorial design was used, achieving 9 experimental runs. The results of the sensory and proximal chemical analysis determined that the best snack formulation was obtained with $90 \%$ of rice content and $10 \%$ of tarwi content; it was also determined that the extrusion temperature was $110^{\circ} \mathrm{C}$. The snack produced had a protein content of $13.11 \%, 4.03 \%$ fiber and a shelf life of 25 days, resulting in a product with a high protein content, high organoleptic acceptance and suitable for consumption.

Keywords: Tarwi flour, rice flour, extrusion, protein, shelf life.

\section{INTRODUCCIÓN}

En la actualidad las personas tienden a consumir alimentos más saludables (Pechey et al., 2021) y viven tan ajetreadas con el trabajo, que no tienen tiempo para preparar sus propios alimentos; por consiguiente, terminan consumiendo alimentos fuera de casa ya sea en restaurantes, snack, supermercados u otros (Sandoval y Camarena, 2011). Los snacks que se encuentran en el mercado, están elaborados principalmente por trigo y maíz, razón por la cual su valor nutritivo es bajo (Schlinkert et al., 2020); limitándose al aporte energético proveniente de carbohidratos y grasas, y existiendo un déficit de proteínas debido a la carencia de algunos aminoácidos esenciales. No obstante, la fabricación de los snacks se está convirtiendo en una industria clave en todo el mundo, por la combinación de la materia prima que se utiliza para su elaboración, lo cual permite el aporte de cantidades significativas de muchos nutrientes (Arora et al., 2020).

Los granos de tarwi (Lupinus mutabilis S.) tienen un elevado contenido de proteína, rica en lisina, un aminoácido esencial presente en cantidades limitadas en otras fuentes vegetales, cuyas propiedades nutricionales supera a las de otras leguminosas, tales como la soya (Rosell et al., 2009; Cortés et al., 2020). Por otro lado, el arroz (Orysa sativa L.) es un alimento básico consumido por muchos países asiáticos y en el Perú (Jala \& Prasad, 2015), el arroz contiene propiedades nutricionales como carbohidratos, almidón, fibra y proteína vegetales (Amagliani et al., 2017). En el ámbito nacional, la desnutrición es uno de los principales problemas de Salud Pública (Sánchez, 2012), la cual es observada por todo el mundo como un retraso en el crecimiento del país (Acosta \& Haddad, 2014). En este sentido, la investigación para realizar la producción de un snack utilizando la mezcla de harinas de tarwi y arroz, teniendo en cuenta los efectos de los parámetros de extrusión, posee una gran relevancia económica y social, debido a los efectos benéficos que este tipo de productos pueden aportar a la población. El objetivo de la investigación fue determinar la mejor temperatura de extrusión en un snack a partir de harinas de tarwi y arroz, realizar la caracterización química y sensorial de los snacks, determinar los porcentajes de fibra y proteínas y la vida útil del snack.

\section{MATERIALES Y MÉTODOS Preparación de las materias primas}

Para la obtención de la harina de arroz, se desarrolló los siguientes procesos: Recepción de la materia prima, selección, clasificación, molienda y tamizado; y para la harina de tarwi se desarrollaron los siguientes procesos: recepción de la materia prima, selección, hidratación, cocción, desamargado, lavado, prensado, secado, molienda y tamizado.

\section{Preparación de las materias primas}

La Tabla 1, muestra las formulaciones de los diferentes tratamientos y temperatura de extrusión 
Tabla 1

\section{Tratamientos de los snacks extruidos}

\begin{tabular}{cccc}
\hline $\begin{array}{c}\text { Tratamientos } \\
(\mathrm{T})\end{array}$ & $\begin{array}{c}\text { Temperatura } \\
\left({ }^{\circ} \mathrm{C}\right)\end{array}$ & $\begin{array}{c}\text { Tarwi } \\
(\%)\end{array}$ & $\begin{array}{c}\text { Arroz } \\
(\%)\end{array}$ \\
\hline T1 & 100 & 12 & 88 \\
T2 & 100 & 10 & 90 \\
T3 & 100 & 8 & 92 \\
T4 & 110 & 12 & 88 \\
T5 & 110 & 10 & 90 \\
T6 & 110 & 8 & 92 \\
T7 & 115 & 12 & 88 \\
T8 & 115 & 10 & 90 \\
T9 & 115 & 8 & 92 \\
\hline
\end{tabular}

\section{Extrusión de las mezclas}

El proceso de extrusión se desarrolló con las siguientes condiciones de trabajo: temperatura de extrusión (100, 110, $\left.115{ }^{\circ} \mathrm{C}\right)$, velocidad de rotación del tornillo $1560 \mathrm{rpm}$, velocidad de alimentación 1 kg/min, velocidad de cuchilla 630 rpm, caudal de agua $19.5 \mathrm{ml} / \mathrm{min}$ y diámetro de la boquilla de salida $0.6 \mathrm{~mm}$.

En la Figura 1, se muestra el flujo de operaciones del proceso de extrusión de la mezcla entre la harina de arroz y tarwi. Se utilizó un diseño factorial multinivel, obteniéndose nueve corridas experimentales los cuales fueron analizados mediante la prueba ANOVA, para comparar los datos procesados, en relación a los porcentajes de proteína y fibra cruda bajo los tres niveles de temperatura; y para comparar la aceptabilidad del color, olor, sabor y textura se utilizó el ANOVA donde se utilizaron 37 repeticiones conforme al siguiente modelo matemático: $Y_{-} i j=\mu+t_{-}$ it $\varepsilon_{-}$ij, donde $Y_{-}$ij se refiere a la observación correspondiente al i-ésimo tratamiento evaluado en la j-ésima repetición; $\mu$ es la media general, t_i es el efecto del i-ésimo tratamiento, con $\mathrm{i}=1,2$, ..., 9, y $\varepsilon_{\text {ij }}$ es el error asociado a la observación Y_ij, conforme a Mongomery y Runger (2003).

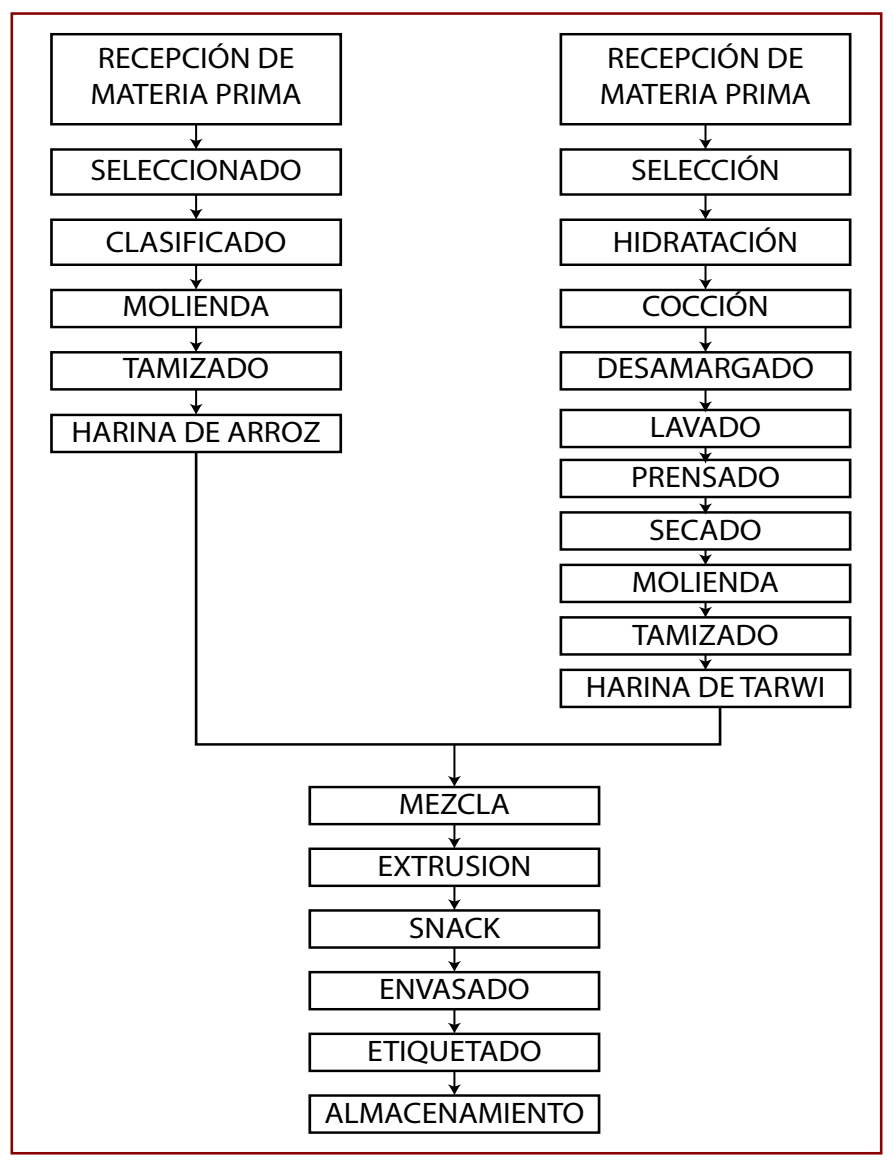

Figura 1. Flujo del procesamiento para la elaboración del snack 


\section{Análisis químico proximal}

La caracterización química de los snacks se efectuó a partir del análisis químico proximal del producto elaborado, determinando los siguientes componentes:

Proteína: se utilizó el método por la N.T.P. 205.005:1979 (2011): cereales y menestras. Se determinaron proteínas totales (Método de Kjeldahl), utilizando 6.25 como factor.

Humedad: se determinó según la N.T.P 205.037:1975 (2011): harinas; el contenido de humedad se determinó por el método de la estufa AOAC.

Grasa: se utilizó el equipo Soxhlet, usando hexano como solvente. Metodología de la Asociación oficial de Químicos Analistas (AOAC) 963.15 2005, método Soxhlet.

Ceniza: se realizó por la incineración de la materia orgánica en una mufla; siguiendo la metodología por la N.T.P 205.038:1975 (2011): harinas.

Carbohidratos: se obtuvo por diferencia restando el $100 \%$ la suma de todos los porcentajes de humedad, ceniza, grasa y proteínas. Metodología para carbohidratos, por diferencia de materia seca (MS-INM) (Collazos, 1993); usando la fórmula: $\%$ Carbohidratos $=100-(\mathrm{H}+\mathrm{C}+\mathrm{G}+\mathrm{P})$

Fibra Dietética Total: se determinó siguiendo la metodología AOAC 15th Edición. U.S.A. (1990). Muestras de alimentos secos y desgrasados son gelatinizadas con a - amilasas térmicamente estables y luego digeridas enzimáticamente con proteasa y amiloglucosidasa para remover la proteína y el almidón. La fibra dietética soluble es precipitada por la adición de etanol, el residuo total se filtra, se lava, se seca y se pesa. En el residuo en duplicado se determina proteína, y en las otras, cenizas.

$\%$ Fibra DieteticaTotal $=$ (Masa del Residuo Masa de la Muestra) * 100

\section{Selección de las formulaciones}

Para seleccionar las formulaciones se utilizaron porcentajes variables de muestras de harinas de tarwi y arroz, conforme a lo presentado en la tabla 1.

\section{Evaluación sensorial}

Se realizó la evaluación sensorial a los nueve tratamientos, utilizando una escala hedónica de 7 puntos (Granito et al., 2010), en donde 1 significó me disgusta mucho y 7 significó me gusta mucho; las cualidades evaluadas fueron: olor, color, sabor y textura, se entregó a cada juez los nueve tratamientos de snacks codificados con números aleatorios de tres cifras. Para la evaluación participaron 37 jueces no-entrenados de la Escuela Académica Profesional de Ingeniería Agroindustrial en la Universidad Nacional del Santa.

\section{Vida Útil}

El análisis de vida útil de los snacks, se determinó mediante análisis sensorial (Corrigan et al., 2012; Elizagoyen, 2019; Sánchez \& Pérez, 2016) por cada semana ya que determina el máximo tiempo en el que el snack es aceptado por los jueces. Los snacks fueron sometidos a pruebas sensoriales con una escala cuantitativa de siete puntos, que va desde me disgusta mucho hasta me gusta mucho. Las pruebas finalizaron cuando los catadores dieron un puntaje igual o menor a 3 , que indicaría que les disgusta levemente los snacks.

\section{RESULTADOS Y DISCUSIÓN Caracterización química proximal del arroz}

En la Tabla 2, se muestra el análisis químico proximal del arroz, determinándose la cantidad de humedad, proteínas, grasa, cenizas y carbohidratos en $100 \mathrm{~g}$ de porción comestible.

\section{Tabla 2}

Caracterización química proximal del arroz en $100 \mathrm{~g}$ de porción comestible

\begin{tabular}{cc}
\hline Componentes & Arroz \\
\hline Humedad & $12.31 \pm 0.51$ \\
Proteínas & $7.34 \pm 0.6$ \\
Grasa & $0.99 \pm 0.09$ \\
Cenizas & $5.03 \pm 0.17$ \\
Carbohidratos & $74.31 \pm 0.87$ \\
\hline
\end{tabular}


En el trabajo de investigación de Amagliani et al., (2017), se determinó que la harina de arroz utilizada tuvo $7.32 \pm 0.04$ de proteínas, $81.1 \pm 0.15$ de carbohidratos, $0.78 \pm 0.0$ de grasa, $1.30 \pm 0.18$ de ceniza y $9.53 \pm 0.02$ de humedad, los cuales concuerdan con la mayoría algunos de los resultados presentados en la Tabla 2, en donde las mayores discrepancias se pueden atribuir al tipo de arroz, al lugar de cultivo del arroz (Carney, 2015) y a las condiciones de análisis.

\section{Caracterización química proximal del tarwi}

En la Tabla 3, se presenta el análisis químico proximal del tarwi, en donde se puede observar las cantidades de proteínas, humedad, grasas, cenizas y carbohidratos en $100 \mathrm{~g}$ de porción comestible.

\section{Tabla 3}

Caracterización química proximal del tarwi en $100 \mathrm{~g}$ de porción comestible

\begin{tabular}{cc}
\hline Componentes $(\mathrm{g})$ & Tarwi \\
\hline Humedad & $4.51 \pm 0.54$ \\
Proteínas & $56.96 \pm 0.43$ \\
Grasa & $23.32 \pm 0.66$ \\
Cenizas & $6.27 \pm 0.39$ \\
Carbohidratos & $8.93 \pm 0.52$ \\
\hline
\end{tabular}

Suca \& Suca (2015), determinaron la composición química proximal del tarwi, reportando 51.1 \% de proteínas, $20.4 \%$ de grasa y $2.4 \%$ de ceniza. Del mismo modo, Niño et al., (2017); determinaron cantidades aproximadas de $8.92 \pm 0.06 \%$ de humedad, $48.06 \pm 0.34 \%$ de proteínas, $22.31 \pm 1.20 \%$ de grasa y $2.68 \pm 0.13$ $\%$ de ceniza en el tarwi. Por su parte, Pantoja et al., (2020), mostraron contenidos de $6.33 \pm 0.15$ $\%$ de humedad, $49.04 \%$ de proteína, $2.85 \pm 0.04$ $\%$ de cenizas, $27.32 \pm 0.25 \%$ de grasa y 11.52 $\%$ de carbohidratos, para la composición de la harina de tarwi precocida. En el trabajo de Carvajal et al., (2016); se observan contenidos de $8.1 \mathrm{~g}$ de humedad, $43.3 \mathrm{~g}$ de proteínas y 3.9 $\mathrm{g}$ de ceniza, como datos promediados para el tarwi. Los valores encontrados en los diversos trabajos de investigación se pueden considerar valores cercanos a los presentados en la Tabla 3, correspondientes a la caracterización química proximal del tarwi. Esta similitud, se puede deber al lugar donde se cultiva, al tipo del suelo, a las condiciones climáticas y a las condiciones de análisis en el laboratorio.

\section{Análisis químico proximal de los snacks}

La Tabla 4, muestra el análisis químico proximal de los nueve tratamientos utilizados en el estudio. 
Tabla 4

Análisis Químico Proximal de los Snacks

\begin{tabular}{cccccccccc}
\hline Componentes & T1 & T2 & T3 & T4 & T5 & T6 & T7 & T8 & T9 \\
\hline Humedad $(\mathrm{g})$ & 1.81 & 1.96 & 2.85 & 2.44 & 1.76 & 2.19 & 1.88 & 3.11 & 1.45 \\
Proteínas $(\mathrm{g})$ & 13.87 & 13.30 & 12.21 & 13.98 & 13.11 & 12.15 & 13.94 & 13.10 & 12.20 \\
Grasa (g) & 0.57 & 0.68 & 1.07 & 1.25 & 1.76 & 0.42 & 1.27 & 0.54 & 0.77 \\
Cenizas (g) & 5.03 & 6.00 & 5.45 & 5.11 & 5.57 & 6.00 & 5.01 & 5.21 & 5.02 \\
Carbohidratos (g) & 77.78 & 77.39 & 76.70 & 77.72 & 77.80 & 79.24 & 79.41 & 78.87 & 80.47 \\
\hline
\end{tabular}

El contenido de proteínas de los nueve tratamientos varió desde 12.20 (T9) a 13.98 (T4), cuyos valores están asociados a la proporción del tarwi y arroz que contienen los snacks.

Se realizó la comparación de las proteínas y la fibra dietética total para los 9 tratamientos del snack (Tabla 5), mediante el ANOVA, mostrando que no hubo diferencia significativa $(p>0.05)$ entre los tratamientos a temperaturas de extrusión de $100^{\circ} \mathrm{C}, 110^{\circ} \mathrm{C}$ y $115^{\circ} \mathrm{C}$. Se han realizado distintos estudios, en donde se demuestra que los procesos de tratamiento térmicos inducen a la desnaturalización de las proteínas y por lo tanto producen un cambio en la estructura física de las mismas; sin embargo, no afectan a la composición de los aminoácidos, en otras palabras, a las propiedades nutricionales (Mejía et al., 2017).

Tabla 5

Cuadrados medios del análisis de varianza (ANOVA) para proteínas (\%) y la fibra dietética total (FDT\%), bajo los tres niveles de temperatura.

\begin{tabular}{cccc}
\hline $\begin{array}{c}\text { Fuentes de } \\
\text { variación }\end{array}$ & Gl & Proteínas & FDT \\
\hline Temperaturas & 2 & 0,00251369 & 0,94414 \\
Error & 6 & 0,76664 & 0,46257 \\
Total (Corr.) & 8 & & \\
\hline
\end{tabular}

La temperatura de extrusión que se aplicó a los snacks no generó pérdidas en la concentración de las proteínas, como se observa en la Figura 2, debido a que las temperaturas generaron niveles de concentraciones de proteínas muy similares. En definitiva, la extrusión conserva los contenidos proteicos en los snacks (Saldanha et al., 2019), debido a que la misma trabaja en tiempos cortos.

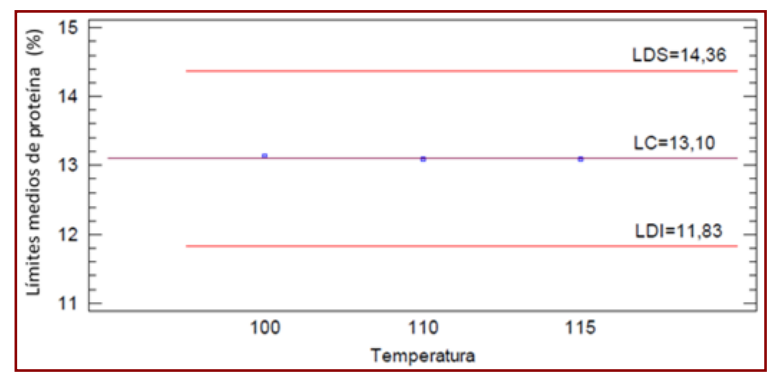

Figura 2. Análisis de medias (ANOM) para Proteínas. LDS: Límite superior, LC: Límite central, LDI: Límite inferior 
Los tratamientos 1, 4 y 7 tienen un mayor porcentaje de proteínas en comparación con los tratamientos 3, 6 y 9 como se observa en la Figura 3, debido a la cantidad de tarwi y arroz que tiene cada tratamiento puesto que ello influye directamente con el contenido de proteínas. Sin embargo, estas diferencias no fueron reflejadas en el ANOVA, lo cual se pudo deber a diferentes factores, dentro de los cuales se pueden resaltar el número de repeticiones utilizadas en el estudio.

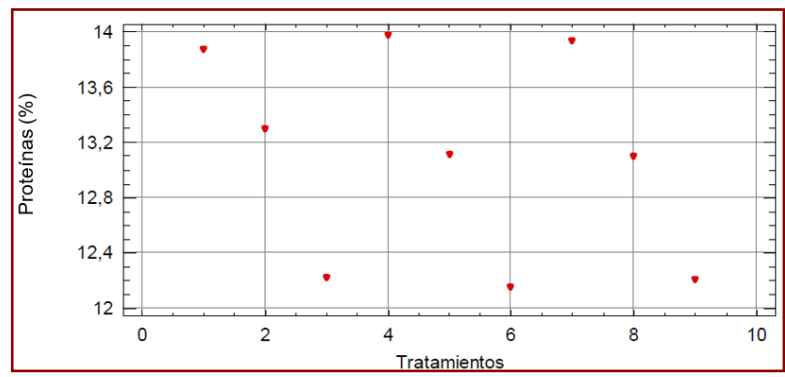

Figura 3. Valores promedios de proteína para los diversos tratamientos.
Ganorkar et al., (2021), determinó que los snacks extruidos con harina de maní, harina de maíz y arroz, presentaron un contenido de proteínas de $13.88 \%$, mientras que los snacks elaborados en este estudio tuvieron un contenido de proteínas de $12 \%$. Estos resultados, demuestran la importancia de haber utilizado la harina de tarwi y arroz en la elaboración de este tipo de productos, debido a sus altos aportes proteicos.

\section{Fibra Dietética Total (\% FDT)}

En el ANOVA que se realizó se mostró que no existe una diferencia estadísticamente significativa entre las medias de la FDT ( $p>0.05$ ) con un nivel del 95\% de confianza (Tabla 5). La determinación de los porcentajes de fibra dietética total para los 9 tratamientos obtenidos por extrusión, se detallan en la Tabla 6.

\section{Tabla 6 \\ Caracterización químico proximal del tarwi en $100 \mathrm{~g}$ de porción comestible, en relación al contenido FDT (\%)}

\begin{tabular}{cccc}
\hline $\begin{array}{c}\text { Tratamientos } \\
(\mathrm{T})\end{array}$ & $\begin{array}{c}\text { Tarwi } \\
(\%)\end{array}$ & $\begin{array}{c}\text { Arroz } \\
(\%)\end{array}$ & $\begin{array}{c}\text { FDT } \\
(\%)\end{array}$ \\
\hline T1 & 12 & 88 & 4.95 \\
T2 & 10 & 90 & 5.19 \\
T3 & 8 & 92 & 4.25 \\
T4 & 12 & 88 & 3.69 \\
T5 & 10 & 90 & 4.03 \\
T6 & 8 & 92 & 3.43 \\
T7 & 12 & 88 & 3.65 \\
T8 & 10 & 90 & 3.18 \\
T9 & 8 & 92 & 5.15 \\
\hline
\end{tabular}

La Figura 4, presenta la influencia de las temperaturas para la fibra y se observa que la temperatura de $110^{\circ} \mathrm{C}$ influye en mayor proporción a la disminución de la fibra sobre los snacks. No obstante, estas variaciones resultaron no significativas desde el punto de vista estadístico.

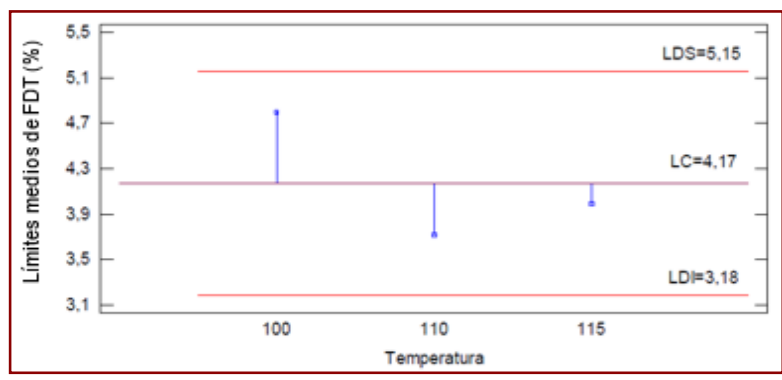

Figura 4. Análisis de medias (ANOM) para FDT LDS: Límite superior, LC: Límite central, LDI: Límite inferior 
En la Figura 5, se detalla la cantidad de fibra de cada snack elaborado y se observa que los mayores niveles los presentaron los tratamientos 2 y 9, mientras que el tratamiento 5 presentó un contenido intermedio, en comparación con los otros snacks, el cual puede ser considerado aceptable.

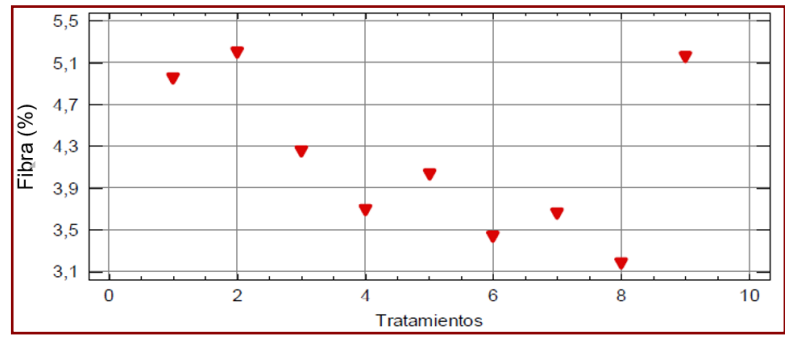

Figura 5. Análisis de medias (ANOM) para FDT

LDS: Límite superior, LC: Límite central, LDI: Límite inferior

La fibra del snack es variada y no hay un patrón que indique la disminución por efecto de la temperatura; además, el ANOVA indicó que no existen diferencias significativas ( $p>0.05$ ) en el contenido de fibra cruda entre los diferentes tratamientos de temperatura. El contenido de fibra varía de acuerdo a las concentraciones de arroz y tarwi que está presente en los nueve tratamientos, el cual resultó en una media de $4,17 \%$, comparado con $7.5 \%$ de fibra obtenida por Camargo et al., (2008) en la extrusión de almidón de yuca.

\section{Análisis Sensorial}

En la Tabla 7, se presentan los análisis de varianza para los atributos sensoriales evaluados en todos los tratamientos incluidos en el estudio, en donde se puede observar diferencias altamente significativas $(p<0.01)$ para los atributos aceptabilidad del sabor y la aceptabilidad de la textura; sin embargo, para la aceptabilidad del color y la aceptabilidad del olor no se observaron diferencias estadísticas significativas ( $p>0.05$ ).

Tabla 7

Cuadrados medios del ANOVA para la evaluación de la aceptabilidad del color (AC), la aceptabilidad del olor (AO), la aceptabilidad del sabor (AS) y la aceptabilidad de la textura (AT) de los nueve tratamientos incluidos en el estudio.

\begin{tabular}{cccccc}
\hline $\begin{array}{c}\text { Fuentes de } \\
\text { variación }\end{array}$ & GI & \multicolumn{4}{c}{ Cuadrados medios } \\
\hline Tratamientos & 8 & 0,8611 & 0,7598 & $5,9917^{* *}$ & $12,5503^{* *}$ \\
Error & 324 & 1,3914 & 1,1165 & 1,6523 & 1,8759 \\
Total (Corr.) & 332 & & & &
\end{tabular}

** indica diferencias significativas al 1\%. 
En la Figura 6, de las medias de los snacks para el color se observa que los tratamientos 1, 3, 7 y 8 tienen mayor aceptabilidad en el análisis; sin embargo, tales diferencias no resultaros estadísticamente importantes, debido a que posiblemente para los jueces el atributo del color no es trascendental.

Algarni et al., (2019) observaron un incremento en el valor de luminosidad $\left(L^{*}\right)$ del color con el incremento en las concentraciones de $\mathrm{L}$. mutabilis en las muestras extruidas.

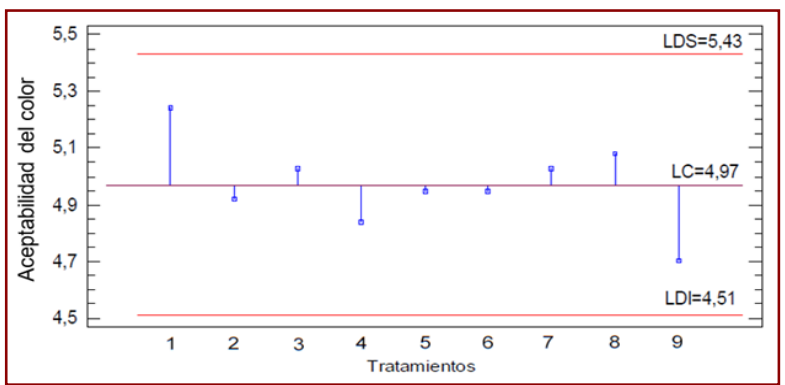

Figura 6. Análisis de medias (ANOM) para Color

LDS: Límite superior, LC: Límite central, LDI: Límite inferior

Algunos estudios revelan que la formación de color durante el proceso de extrusión depende de la temperatura del tratamiento térmico y está directamente relacionada con la composición de la formulación (Teba et al., 2009).

Los resultados del análisis de varianza para el atributo olor (Tabla 7), muestran que no se encontraron diferencias significativas estadísticamente entre las medias de los 9 tratamientos ( $p>0.05)$.

En lo que respecta al parámetro aceptación del olor de los snacks, los tratamientos 2, 4, 5, 6 y 9 (Figura 7), son aceptables en el análisis, demostrando que el contenido de Tarwi y la temperatura de extrusión no influyen en el análisis de olor y además se observa que los tratamientos 1, 4 y 7, que contienen cantidades iguales de Tarwi en su formulación, varían en su aceptabilidad, al igual que los tratamientos 2, 5, y 8 (Figura 7).

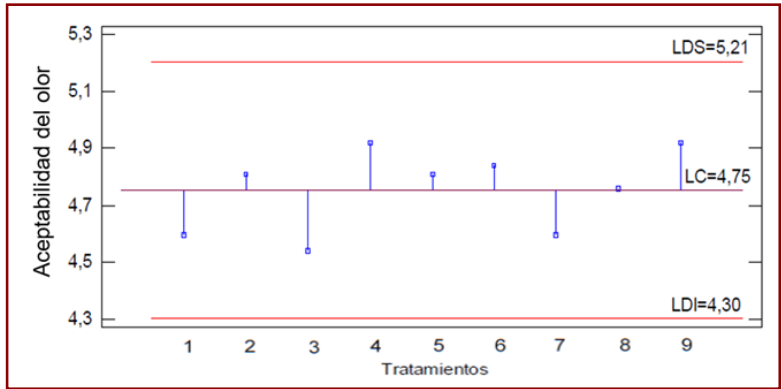

Figura 7. Análisis de medias (ANOM) para Olor

LDS: Límite superior, LC: Límite central, LDI: Límite inferior

El análisis de varianza para el atributo sabor demostró que existen diferencias estadísticas altamente significativas $(p<0.01)$ entre las medias de los 9 tratamientos (Tabla 7). Posiblemente esto se debió a la presencia del tarwi en el snack, el cual les confiere un sabor muy agradable a los snacks, generando tales diferencias, tal como lo referido por Campos et al., (2018).

En la Figura 8, de las medias de los snacks para el sabor se observa que los tratamientos 5, 6 y 9 fueron los más aceptables por los jueces. El sabor más aceptado es el tratamiento 9 con un contenido del $8 \%$ de tarwi y $92 \%$ de arroz, pero el tratamiento 5, que también es significativo por los jueces, contiene 10\% de tarwi y $90 \%$ de arroz. Este último sería el mejor, debido a que representa la mezcla que genera un snack de mejor aceptación.

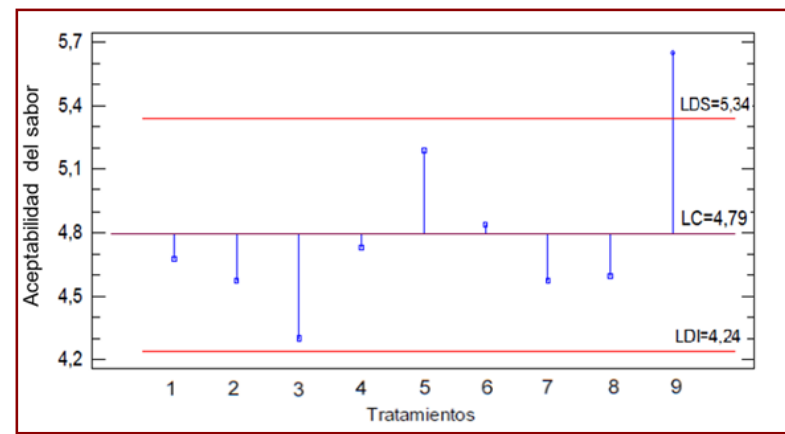

Figura 8. Análisis de Medias (ANOM) para Sabor LDS: Límite superior, LC: Límite central, LDI: Límite inferior

En lo que concierne a la textura de los snacks, el ANOVA reflejó diferencias estadísticas altamente significativas $(p<0.01)$ entre las medias de los nueve tratamientos (Tabla 7). Estos resultados sugieren que al menos dos de los tratamientos analizados difieren en la apreciación de su nivel de textura. 


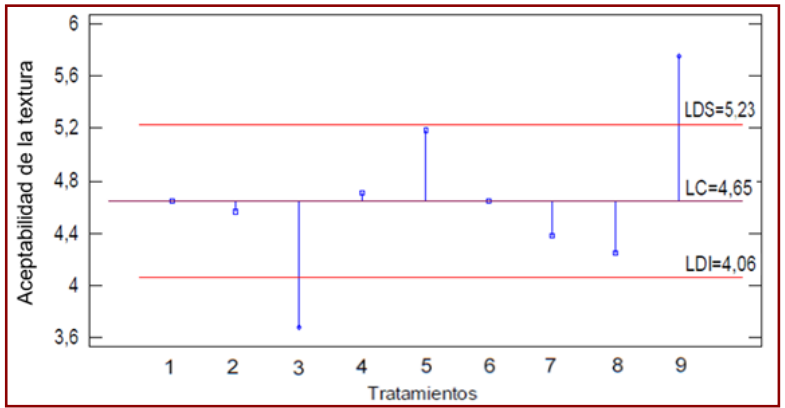

Figura 9. Análisis de medias (ANOM) para Textura LDS: Límite superior, LC: Límite central, LDI: Límite inferior

En la Figura 9, se presentan las medias de los snacks para la textura, donde se puede observar que los tratamientos 4, 5 y 9 resultaron los más aceptados por los jueces. Los resultados además determinaron que hay mayor preferencia por los snacks que contienen mayor cantidad de arroz, debido al tipo de almidón que está presente en el arroz, el cual le confiere al snack una textura más suave y más fácil de masticar. Por lo tanto, desde el punto de vista nutricional y de la aceptación por los jueces, el tratamiento 5 sería el mejor.

\section{Vida Útil}

La determinación del tiempo de vida útil de los snacks se realizó mediante un análisis sensorial. Cada snack obtuvo 25 días de vida útil, el cual corresponde al tiempo estimado en el que el producto conserva sus características organolépticas de calidad, considerándose apto para el consumo.

El análisis sensorial del snack en la semana 1 se observa en la Figura 10; estos snacks obtuvieron una puntación por encima de 6, lo que significa que estos tres tratamientos no fueron bien aceptados por los jueces, quienes mostraron mayor preferencia por el tratamiento 5.

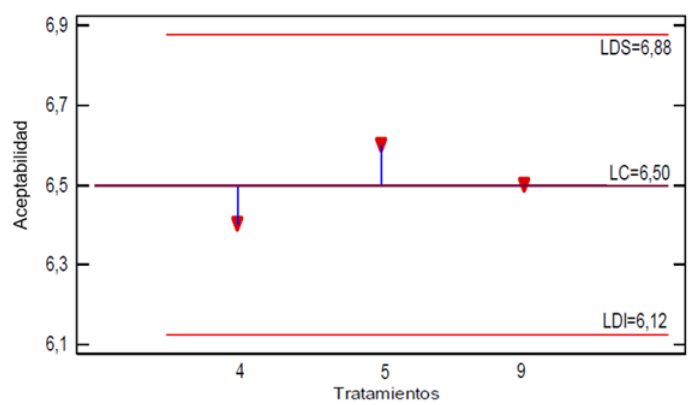

Figura 10. Análisis de medias (ANOM) para Semana 1 LDS: Límite superior, LC: Límite central, LDI: Límite inferior
En la Figura 11, se observa el análisis sensorial del snack efectuado en la semana 2, en donde se puede observar que los tratamientos 4, 5 y 9 mostraron una puntuación alrededor de los 6 puntos. Estos resultados demuestran que estos tres tratamientos tuvieron buena aceptabilidad por los jueces, con mayor preferencia para los tratamientos 4 y 5 .

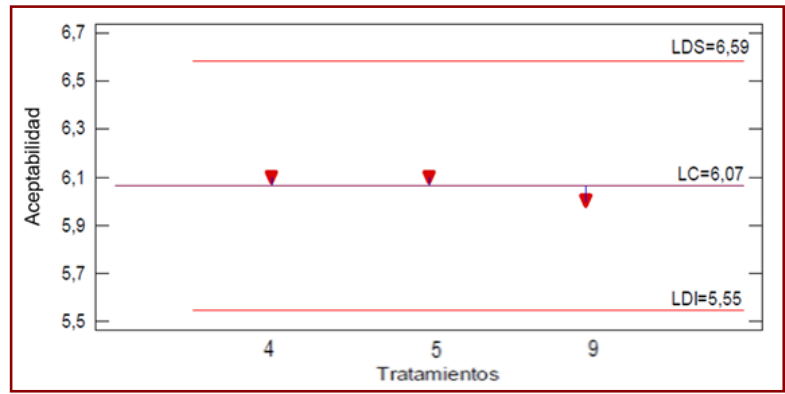

Figura 11. Análisis de medias (ANOM) para Semana 2

LDS: Límite superior, LC: Límite central, LDI: Límite inferior

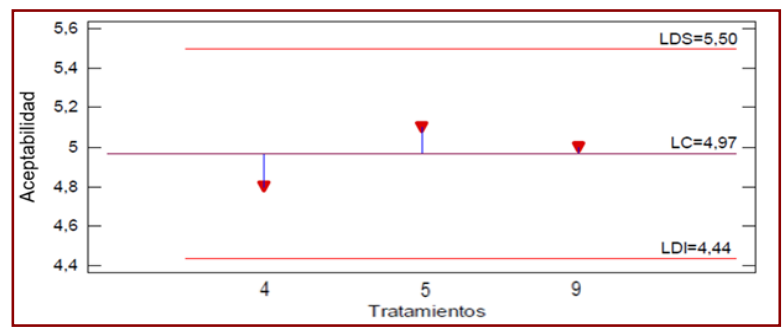

Figura 12. Análisis de medias (ANOM) para Semana 3 LDS: Límite superior, LC: Límite central, LDI: Límite inferior

En la Figura 12, se presentan los resultados del análisis sensorial efectuado para la semana 3 , donde se puede observar que la puntuación dada para los tratamientos 4, 5 y 9 está alrededor de 5 puntos, lo cual quiere decir que a los jueces no le gusta ni les disgustan estos tres tipos de snack, aunque tienen una leve preferencia por el tratamiento 5.

En la semana 4, el análisis sensorial tiende a una puntuación menor de 4 (figura 13), lo que significa que a los jueces le comienza a parecer desagradable los snacks en cuestiones de textura y sabor. Por esta razón, a partir de esta semana se interrumpe el análisis sensorial, ya que el snack no es apetecible para el catador. De acuerdo a Vásquez et al., (2015), la determinación de la vida útil por el análisis sensorial es una de las mejores opciones, dado que el producto se basa en la preferencia del catador y por ello es importante que sea agradable y que tenga preferencia entre los consumidores. 


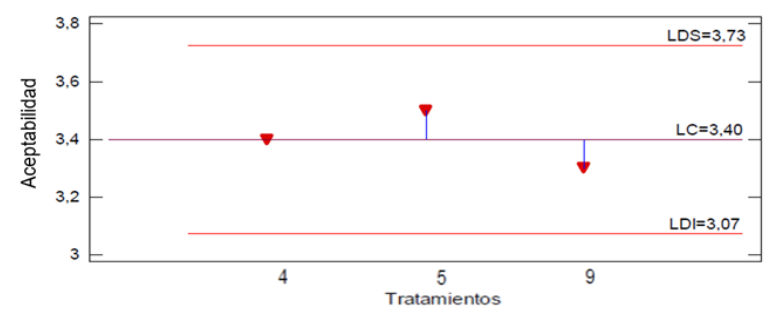

Figura 13. Análisis de medias (ANOM) para Semana 4 LDS: Límite superior, LC: Límite central, LDI: Límite inferior

\section{CONCLUSIONES}

El mejor tratamiento del snack está influenciado por la temperatura de extrusión de $110^{\circ} \mathrm{C}$, por el contenido arroz del $90 \%$ y $10 \%$ de tarwi, debido a su valor nutricional y aceptabilidad por los jueces. La calidad sensorial es influenciada por la temperatura y el tipo de formulación de cada tratamiento.

El tiempo de vida útil determinó que el tratamiento 5 tuvo la mayor durabilidad, con 25 días de vida útil del producto.

\section{REFERENCIAS BIBLIOGRÁFICAS}

Acosta, A. M., \& Haddad, L. (2014). The politics of success in the fight against malnutrition in Peru. Food policy, 44, 26-35. https:// doi.org/10.1016/j.foodpol.2013.10.009.

Algarni; E. H., H. A. Hussien y E. M. Salem. (2019). Development of Nutritious Extruded Snacks. Life Sciences Journal, 16(9), 23-31. Disponible en: http://www.lifesciencesite.com/lsj/ Isj160919/03_35203Isj160919_23_31.pdf.

Amagliani, L., O'Regan, J., Kelly, A. L., \& O'Mahony, J. A. (2017). Composition and protein profile analysis of rice protein ingredients. Journal of Food Composition and Analysis, 59, 18-26. DOl: https:// doi.org/10.1016/j.jfca.2016.12.026

Arora, M., Singhal, S., Rasane, P., Singh, J., Kaur, S., Kumar, V., ... \& Mishra, A. (2020). Snacks and Snacking: Impact on Health of the Consumers and Opportunities for its Improvement. Current Nutrition \& Food Science, 16(7), 1028-1043. DOI: https://doi. org/10.2174/1573401316666200130110357
Bhat, N. A., Wani, I. A., Hamdani, A. M., \& Gani, A. (2019). Effect of extrusion on the physicochemical and antioxidant properties of value added snacks from whole wheat (Triticum aestivum L.) flour. Food chemistry, 276, 22-32. DOl: https:// doi.org/10.1016/j.foodchem.2018.09.170

Camargo, KFD, Leonel, M. y Mischan, MM (2008). Producción de galletas extruidas a partir de polvo ácido con fibras: efecto de los parámetros operativos sobre las propiedades físicas. Ciencia y tecnología de losalimentos, 28(3),586-591.DOI:https://doi. org/10.1590/S0101-20612008000300013

Campos, D., Chirinos, R., Ranilla, L. G., \& Pedreschi, R. (2018). Bioactive potential of andean fruits, seeds, and tubers. In Advances in food and Nutrition Research, 84, 287343). Academic Press. DOl: https:// doi.org/10.1016/bs.afnr.2017.12.005

Carney, J. A. (2015). El origen africano del cultivo del arroz en Las Américas. Asclepio, 67(1), 077. DOl: https:// doi.org/10.3989/asclepio.2015.03

Carvajal-Larenas, FE, Linnemann, AR, Nout, MJR, Koziol, M. y van Boekel, MAJS (2015). Lupinus mutabilis: Composición, Usos, Toxicología y Desamor. Revisiones críticas en ciencia de los alimentos y nutrición, 56 (9), 1454-1487. DOl: 10.1080 / 10408398.2013.772089.

Cortés-Avendaño, P., Tarvainen, M., Suomela, JP. Glorio, P., Yang, B., Carrasco, R. (2020). Profile and Content of Residual Alkaloids in Ten Ecotypes of Lupinus mutabilis Sweet after Aqueous Debittering Process. Plant Foods Human Nutrition 75, 184-191.DOI: https:// doi.org/10.1007/s11130-020-00799-y

Corrigan, V., Hedderley, D., \& Harvey, W. (2012). Modeling the shelf life of fruit-filled snack bars using survival analysis and sensory profiling techniques. Journal of sensory studies, 27(6), 403-416. DOl: https://doi.org/10.1111/joss.12006 
Elizagoyen, E. S. (2019). Factores que influyen sobre la vida útil sensorial de productos alimenticios: almacenamiento en el hogar, fecha devencimiento, tipo de producto, perfil del consumidor y entorno de evaluación (Doctoral dissertation, Universidad Nacional de La Plata (UNLP). Disponible en: http:// sedici.unlp.edu.ar/handle/10915/79617

Ganorkar, P. M., Shah, M. V., Kamariya, J. C., \& Mathew, Y. (2021). Development of Groundnut Meal Incorporated Rice-Corn Flour Based Extruded Snack-Food. Agricultural Research, 10(2), 324-333. DOl: https://doi. org/10.1007/s40003-020-00495-y

Granito, M., Valero, Y., \& Zambrano, R. (2010). Baked product development based fermented legumes and cereals for schoolchildren snack. Archivos latinoamericanos de nutricion, 60(1), 85-92. Disponible en: https:// www.scopus.com/inward/record.uri?eid=2s2.0-78650489740\&partner|D $=40 \&$ md5 $=71 \mathrm{dccf0} 2 \mathrm{bdaec} 4 \mathrm{fd} 5 \mathrm{cc} 91$ eea $5 \mathrm{c} 142 \mathrm{~b} 1 \mathrm{a}$

Jala, R. C. R., \& Prasad, R. B. N. (2015). Rice Bran Lecithin: Compositional, Nutritional, and Functional Characteristics. In: Polar Lipids (pp. 35-55). Elsevier. DOI: https://doi. org/10.1016/B978-1-63067-044-3.50006-6

Mejía-López, Ana, Rodas, Sonia, \& Baño, Darío. (2017). La desnaturalización de las proteínas de la leche y su influencia en el rendimiento del queso fresco. Enfoque UTE, 8(2), 121-130. DOI: https:// doi.org/10.29019/enfoqueute.v8n2.162.

Mongomery, D. C. y Runger, G. C. (2003). Probabilidad y estadística aplicada a la Ingeniería. 2da. Ed. Editorial Limusa Wiley. México D. F. 486 p. Disponible en: https:// kupdf.net/queue/probabilidad-y-estadiacute-stica-aplicadas-a-la-ingenier-iacutea-montgomery-runger-2-ordm-edicioacute-n-cap-1-8_590c6591dc0d601f 59959 e d $0_{\text {_ }}$ p d f? q u e u e _ id $=1 \& x=1623023464 \& z=M T g \times L j E 3 N i 4 x N j c u O T Q=$
Niño, R. M. V., Nogueroles, A. Z., \& Vegas, C. (2017). Efecto del pH y cloruro de sodio sobre las propiedades funcionales de harina de semillas de lupinus mutabilis "tarwi" variedad criolla. Agroindustrial Science, 7(1), 49-55. DOl: https://doi. org/10.17268/agroind.science.2017.01.05

Pantoja-Tirado, L., Prieto-Rosales, G., \& Aguirre Vargas, E. (2020). Caracterización de la harina de quinua (Chenopodium quinoa Willd.) y la harinadetarwi(Lupinus mutabilisSweet) para su industrialización. REVISTA TAYACAJA, 3(1). DOI: $\quad$ https://doi.org/10.46908/rict.v3i1.72

Pechey, R., Sexton, O., Codling, S., \& Marteau, T. M. (2021). Impact of increasing the availability of healthier vs. less-healthy food on food selection: a randomised laboratory experiment. BMC public health, 21(1), 1-12. DOl: https:// doi.org/10.1186/ISRCTN34626166

Rosell, C. M., Cortez, G., \& Repo-Carrasco, R. (2009). Breadmaking use of andean crops quinoa, kañiwa, kiwicha, and tarwi. Cereal chemistry, 86(4), 386-392. DOI: https:// doi.org/10.1094/CCHEM-86-4-0386

Saldanha do Carmo, C., Varela, P., Poudroux, C., Dessev, T., Myhrer, K., Rieder, A.,... Knutsen, SH (2019). El impacto de los parámetros de extrusión en las propiedades fisicoquímicas, nutricionales y sensoriales de snacks expandidos a partir de fracciones de guisante y avena. LWT, 112, 108252. DOl: 10.1016 / j.lwt.2019.108252

Sánchez-Abanto, José. (2012). Evolución de la desnutrición crónica en menores de cinco años en el Perú. Revista Peruana de Medicina Experimental y Salud Publica, 29(3), 402-405. Disponible en: http://www.scielo.org.pe/ scielo.php?script=sci_arttext\&pid=S1726$46342012000300018 \&$ lng=es\&tlng=es.

Sánchez-González, J. A., \& Pérez Cueva, J. A. (2016). Sensory shelf life of mantecoso cheese using accelerated testing. 
Scientia Agropecuaria, 7, 215-222. DOl: 10.17268 /sci.agropecu.2016.03.08

Sandoval Godoy, S. A., \& Camarena Gómez, D. M. (2011). Comportamiento alimentario y perfil de consumo de los sonorenses: el caso de las comidas internacionales. Región y sociedad, 23(50), 185-213. Disponible en: http://www.scielo.org.mx/ scielo.php?script=sci_arttext\&pid=S1870$39252011000100008 \& \operatorname{lng}=e s \& \operatorname{lng}=$.

Schlinkert, C., Gillebaart, M., Benjamins, J., Poelman, M., \& de Ridder, D. (2020). The snack that has it all: People's associations with ideal snacks. Appetite, 152, 104722. DOI: https:// doi.org/10.1016/j.appet.2020.104722

Suca, G., \& Suca, C. (2015). Potencial del tarwi (Lupinus mutabilis Sweet) como futura fuente proteínica y avances de su desarrollo agroindustrial. Revista Peruana De Química E Ingeniería Química, 18(2), 55-71. Disponible en: https:// revistasinvestigacion.unmsm.edu.pe/ index.php/quim/article/view/11791.

Teba, C.S., Ascheri, J.L.R. and Carvalho, C.W.P. (2009). Efeito dos parâmetros de extrusão sobre as propriedades de pasta de massas alimentícias pré-cozidas de arroz e feijão. Alimentos e Nutrição, 20, 411-426. Disponible em: http:// serv-bib.fcfar.unesp.br/seer/index. php/alimentos/article/view/1135/838.

Vásquez-Villalobos, V., Vásquez Angulo, J., \& Méndez Reyna, E. (2015). Nuevo método para determinar vida útil sensorial utilizando lógica difusa: caso corazones de alcachofa (Cynara scolymus L.) marinadas en conserva. Scientia Agropecuaria, 6(2), 99-109. DOl: http://dx.doi. org/10.17268/sci.agropecu.2015.02.02 